\title{
Design and simulation of a multibeamlet injector for a high cur rent accelerator
}

\author{
David P. Grote* \\ LLNL, University of California, Livermore, California 94550 \\ and Virtual National Laboratory for Heavy-Ion Fusion \\ Enrique Henestroza and Joe W. Kwan \\ LBNL, University of California, Berkeley, California 94720 \\ and Virtual National Laboratory for Heavy-Ion Fusion \\ (Received 9 September 2002; published 8 January 2003)
}

\begin{abstract}
A multibeamlet approach to a high current ion injector, whereby a large number of beamlets are accelerated and then merged to form a single beam, offers a number of potential advantages over a monolithic single beam injector. These advantages include a smaller transverse footprint, more control over the shaping and aiming of the beam, and more flexibility in the choice of ion sources. A potential drawback, however, is a larger emittance. In this paper, we seek to understand the merging of the beamlets and how it determines the emittance. When the constraints imposed by beam propagation physics and practical engineering issues are included, the design is reduced to a few free parameters. We describe the physics design of a multibeamlet injector and produce a design for an example set of parameters. Extensive use of 2D and 3D particle simulations was made in understanding the injector. Design tolerances and sensitivities are discussed in general and in relation to the example.
\end{abstract}

PACS numbers: 41.85.Ar, 41.75.Ak, 29.27.Ac, 29.27.Bd

\section{INTRODUCTION}

The requirement of increased particle beam luminosity has driven the need for beam sources which simultaneously have high current and low emittance. The practical limits in single beam sources, however, limit the brightness that can be obtained. We demonstrate here that the limits on brightness can be circumvented by utilizing a multibeamlet injector, showing that the scaling of multibeamlet injectors allows better sources. We also show that a multibeamlet injector can have other practical advantages, such as better control over the beam.

The traditional approach in a single beam ion source is a Pierce gun geometry. See, for example, [1]. The beam is created in a diode and accelerated to the desired energy and then must be matched into the subsequent transport channel. The current density in an ideal parallel plane diode is limited by the Child-Langmuir relation,

$$
J_{\mathrm{CL}} \propto \frac{V^{3 / 2}}{d^{2}},
$$

where $V$ is the voltage drop across the diode whose length is given by $d$. The total current is then $I_{\mathrm{CL}}=A J_{\mathrm{CL}}$, where $A$ is the area of the emitting surface. Minimizing geometric aberrations limits the maximum value of $\left(A / d^{2}\right)$. Furthermore, voltage breakdown limits the voltage to be proportional to $\sqrt{d}$ for $d$ greater than a centimeter $[2,3]$. These constraints result in a total current that varies inversely with the current density, $I_{\mathrm{CL}} \propto J_{\mathrm{CL}}^{-3 / 5}$ - the higher the desired current, the lower the current density, and therefore the larger the size of the emitter. A solution to this scaling problem is to use multiple beams, so $I_{\text {Total }} \propto N J_{\mathrm{CL}}^{-3 / 5}$, where $N$ is the number of beams.
Holding the total area of the emitter, $A_{\text {Total }} \propto N A$, to be constant then leads to an increasing current density with increasing total current, since $I_{\text {Total }}=N I_{\mathrm{CL}}=$ $N A J_{\mathrm{CL}}$. The beams are subsequently merged to form a single beam which propagates into the transport channel. Note that the scalings used here are independent of the particle mass.

A major design requirement is the production of a beam with low emittance. When the beamlets merge (henceforth the individual beams will be referred to as "beamlets" and the merged whole as the "beam") the empty phase space between the beamlets can become entrained in the beam, possibly resulting in a significantly large emittance. The final emittance can be estimated by assuming that all of the available free energy goes into emittance. The free energy here is defined as the difference between the self-field energy of the beamlet configuration and that of a single, uniform beam with the same perveance and rms radius as the combined beamlets. The free energy is usually normalized by the selffield energy inside the equivalent uniform beam. The final emittance has been calculated for a configuration of beamlets in concentric circles assuming that the total beam is matched, centered, and properly aimed [4].

$$
\frac{\varepsilon_{x \mathrm{f}}^{2}}{\varepsilon_{x \mathrm{i}}^{2}} \approx \frac{\left\langle X^{2}\right\rangle+\left\langle Y^{2}\right\rangle}{2\left\langle X^{2}\right\rangle}\left(1+\frac{Q U_{n}}{8\left\langle X^{\prime 2}\right\rangle}\right) .
$$

Here, $\varepsilon_{x \mathrm{f}}$ and $\varepsilon_{x \mathrm{i}}$ are the final and initial emittances, $\left\langle X^{2}\right\rangle$, $\left\langle Y^{2}\right\rangle$, and $\left\langle X^{\prime 2}\right\rangle=\left\langle Y^{\prime 2}\right\rangle$ are the mean square beam sizes and velocities, $Q$ is the total perveance, and $U_{n}$ is the normalized free energy. Defining the occupancy factor $\eta=2 a / \Delta_{r}$ where $a$ is the beamlet radius and $\Delta_{r}$ is the 
center-to-center spacing of the concentric rings of beams, $(0<\eta<1)$, an approximate expression for the normalized free energy was written in terms the occupancy factor and the number of beams, $N$.

$$
U_{n} \approx \frac{4}{N}\left(\frac{3}{4}-\ln 3-\ln \eta+\frac{3}{8} \eta^{2}\right)
$$

These relations are expected only to give qualitative scalings since the beam conditions are outside the range of applicability. There is no focusing in the merging region for the beam to be matched to and the beamlets will be converging and accelerating.

These relations do show that the emittance growth after the merge can be reduced by the following.

(i) Increasing the energy at the merge location, which decreases $Q$.

(ii) Decreasing the spacing between beamlets, which increases $\eta$ and so decreases $U_{n}$ (note that the $\ln \eta$ term dominates).

(iii) Increasing the number of beamlets, which also decreases $U_{n}$.

Use of these three criteria leads to a design wherein a large number of small beamlets are first accelerated in separate channels which are placed as close to each other as engineering constraints will allow. In the next section, the details of the design of such an injector are presented and an example given. In the last section, analytic estimates of the merged emittance are made using energy conservation.

\section{INJECTOR DESIGN}

The basic layout of the injector consists of two sections, the preaccelerator and the merging region; see Fig. 1. In the merging region, the beamlets are both merged and aimed so that the combined beam enters the transport channel. The merging region can also be used to further accelerate the beam up to the design value of the transport channel. The ability to aim the beamlets can be exploited by arranging for the combined beam to enter the channel exactly matched to it. In an alternating gradient, quadrupole focusing channel, for example, if the first quadrupole is half-length, then the combined beam is matched if it has zero convergence and the correct transverse dimensions. This offers a substantial advantage by eliminating the traditional matching section and its associated problems. The matching section typically must transversely compress a large beam to fit into the smaller transport channel. The beam manipulations to do the compression often have been found to cause emittance growth. Eliminating the matching section also can reduce the transverse footprint since, in the traditional case, matching a round beam to an elliptical beam in the alternating gradient transport channel can require large excursions in the beam envelope [5].

For the preaccelerator, a novel Einzel lens design is used, a series of alternating gradient Einzel lenses with a net accelerating voltage. The voltage differences between aperture plates alternate in sign and there is a net voltage drop along the column. The beamlets are accelerated in parallel channels. The Einzel lenses confine the beamlets transversely against space-charge repulsion and thermal expansion. The focusing strength of such lenses diminishes with increasing beam energy however, so the length of the column is limited. This limit must be balanced with the desirability of merging at higher energy. The beamlet size, for a given beamlet current, is constrained by the focusing strength of the Einzel lenses, which depends on the voltage differences and distance between plates, the aperture radius, and the beam energy.

To minimize emittance growth from the merging, the beamlet channels are placed as close to each other as possible. Some a minimal amount of material is needed between the apertures for mechanical stability, preventing excessive warping from mechanical and thermal stresses. Also, the beamlets must be far enough away from each other, compared to the aperture plate separation, so that the electric forces between the beamlets are shielded and are small. Transverse offsets of the apertures (due to construction errors and misalignments) give the

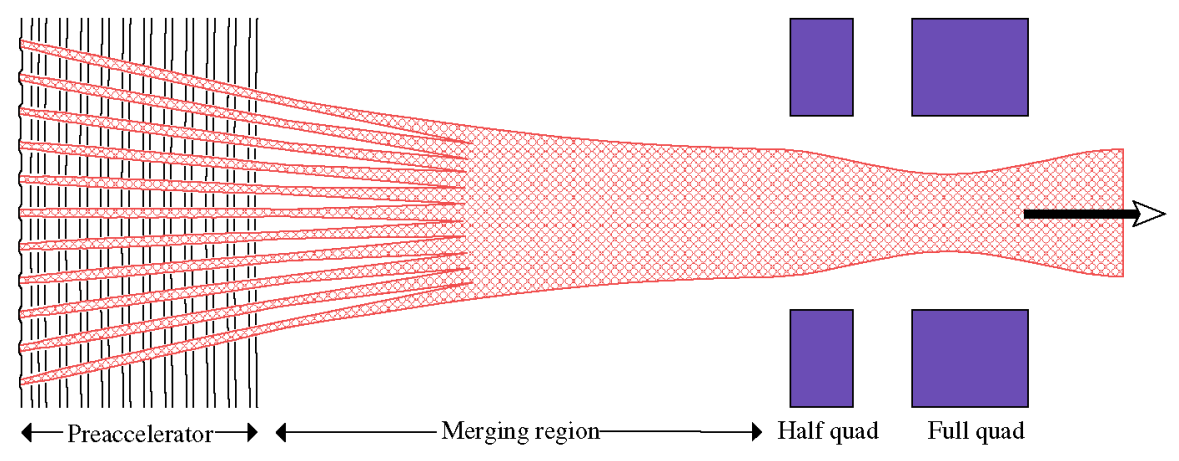

FIG. 1. (Color) Layout of the multibeamlet injector. Each of the beamlets is accelerated independently and then merged. The total beam is matched to the subsequent transport lattice. 
beamlets transverse kicks, moving them off axis. The apertures must be large enough so that, with the beamlets off axis due to the errors, they do not touch the apertures.

The end conditions of the merging section are given by the matched beam parameters in the transport channelthe current, energy, and beam size and divergence. With fixed rms beam size at the start of the merge (averaging over all of the beamlets), and fixed beam size and divergence at the start of the transport, the transverse rms envelope solution for the propagation between the two points is unique. This sets unique values for the length of the merging region and the beam rms convergence at the start of the region. Note that the unique solution depends on any acceleration in the merging region. The unique solution can be found by following the beam backward from the start of the transport channel-the transverse rms envelope expands freely to match to the size of the beamlet arrangement of the final plate of the accelerating column. Note that the length of the merging region increases with the merging energy. This puts a lower limit on the merging energy - with decreasing merging energy, the increasing acceleration needed to get to the design energy of the transport channel must happen over a decreasing merging length and is limited by voltage breakdown constraints.

Given the constraints, there are two primary free parameters in the design: the number of beamlets and the energy at which the beamlets are merged. The number of beamlets, $N$, along with the transport channel current, gives the current per beamlet. The focusing strength of the Einzel lenses, as well as the maximal current density obtainable from emitters, constrains the beamlet radius for that current. The beamlet radius, the clearance space between the beamlet and aperture, and the amount of metal between apertures all then determine the transverse size of the configuration of $N$ beamlets. The unique solution to the envelope equation in the merging region then provides the length of the region, as well as the beamlet aiming angle at the final aperture plate. This nearly completes the design.

There is flexibility with some parameters, allowing optimization of the final emittance of the merged beam. For example, the transverse convergence of the individual beamlets as they exit the accelerating column can be varied. Ideally, if the individual convergences of the beamlets are the same as the aiming convergence at the point where the beamlets just touch each other, the emittance would be minimized. In the transverse phase space, this can be visualized as closing the blinds on a window, where the slats lay against each other to form a nearly flat surface with no holes. However, because of the dynamics, including space-charge effects, the optimal convergence for a given configuration is different from that value and is difficult to estimate analytically. It is straightforward, though, to compute specific cases via simulation.
The emittance of the individual beamlets at the source is also not constrained. The emittance depends mostly on the type and temperature of ion source used. Two potential sources are surface ionization and plasma sources [1], which have different ion temperatures. As will be shown in the example, for small to modest source temperatures, the final emittance is only weakly dependent on the source emittance. This insensitivity can allow much more flexibility in the choice of ion source. We assume here that there will be negligible emittance growth as the beamlets propagate through the accelerating column, which is borne out by simulation.

\section{INJECTOR EXAMPLE}

As an example, a multibeamlet injector suitable for an intermediate term facility for heavy-ion fusion is designed. The principal parameters desired at the beginning of the transport are given in Table I. The most stringent design parameter is the low emittance. The goal of the design will be keeping the emittance as low as possible given the other constraints on the design. The accelerator will be a multibeam accelerator with the beams accelerated side by side. Note that cost optimization requires the beams to be packed closely together. In an electric transport lattice, the beams share electrodes with their neighbors. Ideally, the beam lines would follow a straight line path from the injector into the transport line, thus greatly reducing the complexity (no bends) and the cost. The transverse footprint of the injector would then have to be less than or equal to the transverse size of the transport line. In the example case here, this will be roughly $7 \mathrm{~cm}$ in radius.

As a reference case, we choose 91 beamlets (five rings plus the center) merging at $1.2 \mathrm{MeV}$. See Fig. 2(a) for the layout of the beamlets. For a system of beamlets as assumed in deriving Eq. (3), 100 beamlets is just beyond the "knee" in the curve where more beams give little further decrease in the merged emittance. When using the configuration of concentric rings of beamlets plus the one at the center, the number of beamlets is given by $N=$ $1+3 M(M+1)$, where $M$ is the number of rings around the center beamlet. With $M=5$, there are 91 beamlets. The beamlet radius, $r$, can be estimated by balancing the focusing force of the Einzel lens with the space-charge

TABLE I. Parameters required at the entrance to the example transport lattice.

\begin{tabular}{lc}
\hline \hline Ion & $K^{+}$ \\
Generalized perveance & 0.001 \\
$\sigma_{0}, \sigma$ & $65^{\circ}, 7^{\circ}$ \\
Energy & $1.6 \mathrm{MeV}$ \\
Current & $0.57 \mathrm{~A}$ \\
Emittance & $\leq 1 \pi \mathrm{mm} \mathrm{mrad}$ \\
Beam major and minor radii & $2.0,1.3 \mathrm{~cm}$ \\
\hline \hline
\end{tabular}



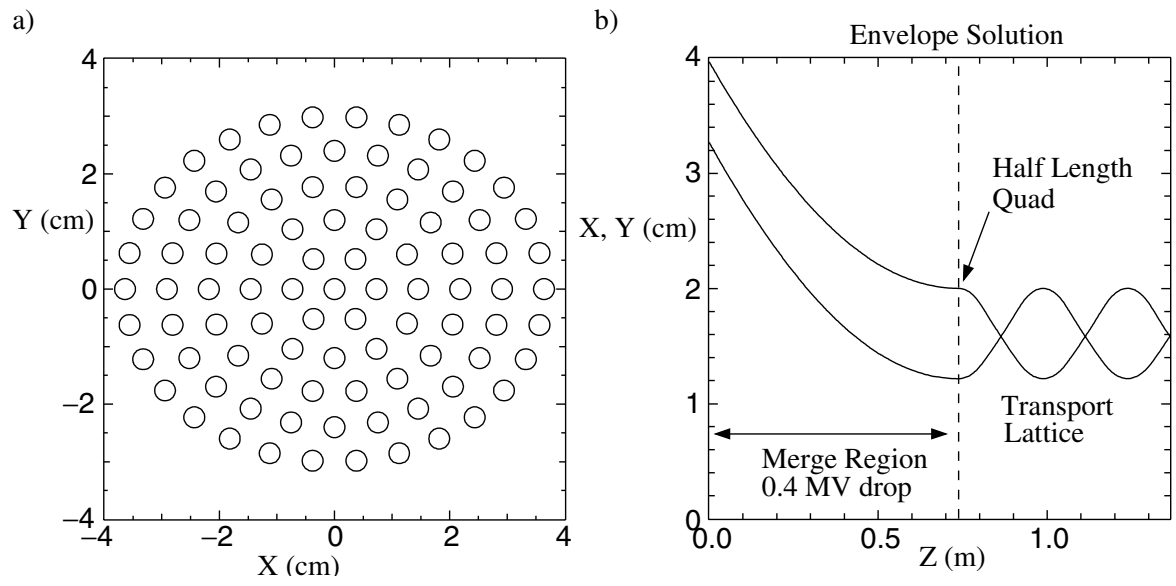

FIG. 2. The configuration of apertures at the last accelerating plate. Each aperture has a radius of $2 \mathrm{~mm}$. The elliptical arrangement allows the combined beam to be exactly matched into the quadrupole transport channel.

force [6]:

$$
r^{2} \approx \frac{9}{8 \pi \varepsilon_{0}} I \sqrt{\frac{m}{2 q} V}\left(\frac{L}{\Delta V}\right)^{2}
$$

Here $I$ is the current per beamlet, $V$ is the beamlet energy in volts, $\Delta V$ is the voltage difference between plates, $L$ is the plate separation, and $m$ and $q$ are the mass and charge of the particles. With the values for this example at the end of the accelerating column, where $V=1.2 \mathrm{MeV}$, and a voltage gradient, $\Delta V / L$, of $100 \mathrm{kV} / \mathrm{cm}$, gives $r \sim$ $1 \mathrm{~mm}$. With a clearance of $1 \mathrm{~mm}$ between the beamlet and aperture, the aperture radius is $2 \mathrm{~mm}$. Another parameter is the distance between beamlets, which is constrained by the need for mechanical rigidity of the aperture plates as well as the need for electrostatic shielding between the beamlets. It was felt that $2 \mathrm{~mm}$ was a reasonable value, giving enough material to support the array of aperture holes. This gives a total of $6 \mathrm{~mm}$ centerto-center ring separation. The design of the preaccelerator column is described in the next section. The design of the merging section, discussed immediately below, assumes beamlets with those parameters.

\section{A. Merging region}

The transverse envelope equation solver in WARP [7] was used to find the unique solution in the merging region. The parameters fixed by the design are the minimum rms radius (set by the minimum beamlet-to-beamlet spacing), the acceleration (up to the desired total injection energy), and matching into the transport lattice. The beam size is held fixed in one direction to the minimum rms radius. In the other, it is allowed to increase, giving an elliptical arrangement. The free parameters are then the initial convergence angle in the two planes, the major radii of the ellipse, and the length of the merging region. For the case with 91 beamlets, $6 \mathrm{~mm}$ beamlet-to-beamlet spacing, and 1.2 MeV merging energy, the envelope solution gives major and minor radii of 39.7 and $32.7 \mathrm{~mm}$, convergence angles of 51.4 and $53.1 \mathrm{mrad}$, and a length of the merging region of $0.7378 \mathrm{~m}$. The configuration of beamlets is shown in Fig. 2(a). Figure 2(b) shows the envelope solution in the merging region and into the first few quadrupoles. The beamlets along each transverse axis converge toward a point. However, with the elliptical layout, the focus is astigmatic, the focal plane for the two axes are different. The convergence angles also give the aiming angles of the accelerating columns. Because of the aiming, each aperture plate is curved, with radii of curvature (different in the two transverse axes) equal to the outer semiaxes of the beamlets divided by the convergence angle, which are approximately 0.77 and $0.62 \mathrm{~m}$, respectively, for the last plate of the preaccelerator column.

To characterize the design, transverse slice simulations of the merging region and following transport were carried out using the WARPXy code [7]. For most of the parameters, there is a monotonic change in emittance as the parameter is varied. Other constraints, such as voltage breakdown, place limits on the parameters - the goal is to minimize the emittance within the constraints. The individual beamlet convergence angle at the start of the merge region, however, has, for a given set of the other parameters, an optimal value minimizing the emittance. For this reason, for each set of parameters studied, a range of convergences was examined to find the minimum. Some of the data presented here are graphs of emittance versus convergence angle. Note that for these simulations, it was assumed that an accelerating column could be designed which produced beamlets with the correct parameters.

Some care was needed in setting the resolution of the slice simulations. It was found that fairly high resolution was needed to reach numerical convergence. The 

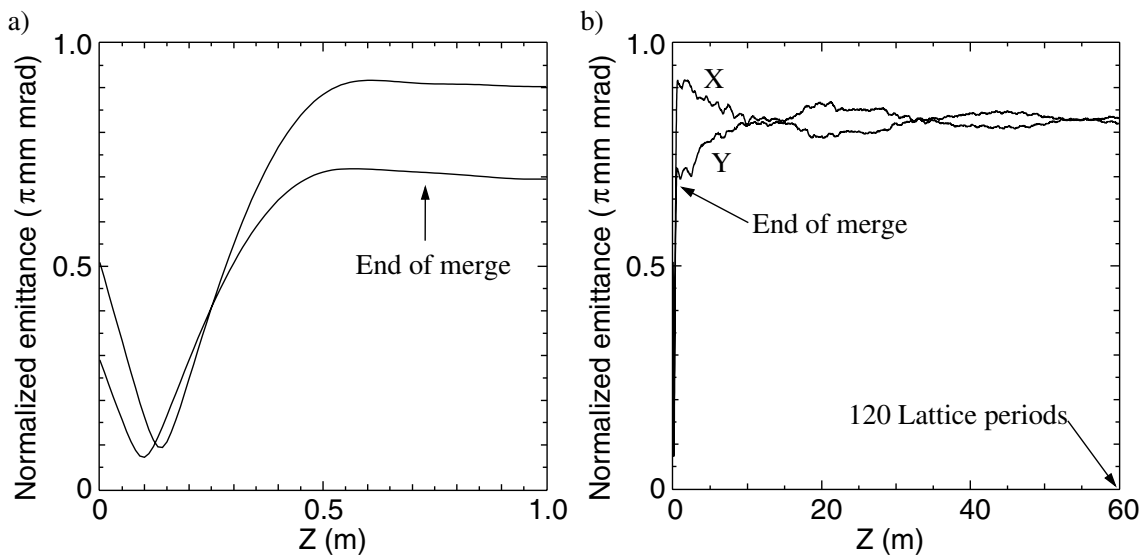

FIG. 3. Long term emittance behavior after merging. (a) Detail of the emittance over the first meter. (b) History over 120 lattice periods.

individual beamlets needed to be well resolved. In the simulations discussed here, the mesh size was $512 \times 512$, giving about ten grid cells across the radius of each beamlet. To reduce particle noise, 20000 particles were used for each beamlet. Higher resolution and particle number does not significantly alter the result. The bulk of the simulations were carried out on the IBM SP at NERSC and typically required $15 \mathrm{~min}$ each on 16 processors.

As the beamlets merge, the overall emittance changes rapidly, reaching its final value just as the beam enters the first quadrupole, showing a rapid mixing of the beamlets. Figure 3 shows the emittance history of that case with merging at $1.2 \mathrm{MeV}$, beamlet radii of $1.5 \mathrm{~mm}, 0.5 \mathrm{~mm}$ clearance, and convergence of $-3.5 \mathrm{mrad}$. Simulations covering several hundred additional lattice periods show no further emittance growth. The $x$ and $y$ emittances initially rise to different values because of the elliptical arrangement of beamlets, differing by as much as $50 \%$ in some cases. As the beam propagates further, the emittances equilibrate to approximately the average value in a few undepressed betatron periods. Others have shown that in some cases when a small number of beamlets are merged, the initial pattern reappears, the location depending on the depressed phase advance [8]. Such reappearance has not been observed in the simulations carried out here.

The merging of the beamlets creates complex spacecharge waves that propagate across the beam and mix. Figure 4 shows contours of density at several locations in configuration space $(x-y)$ and in phase space $\left(x-x^{\prime}\right)$. They show that the individuality of the beamlets is quickly lost, leaving a nearly uniform beam with short wavelength perturbations. Those perturbations diminish after several depressed betatron periods and do not cause any further increase in emittance. The merging process does produce a small halo population. In the simulation of the typical case, after propagating about one depressed betatron period beyond the merge, $10 \mathrm{~m}$ in this case, about $1 \%$ of the beam is in the halo.

The merged emittance is examined, varying the two principal parameters: the number of beamlets and the

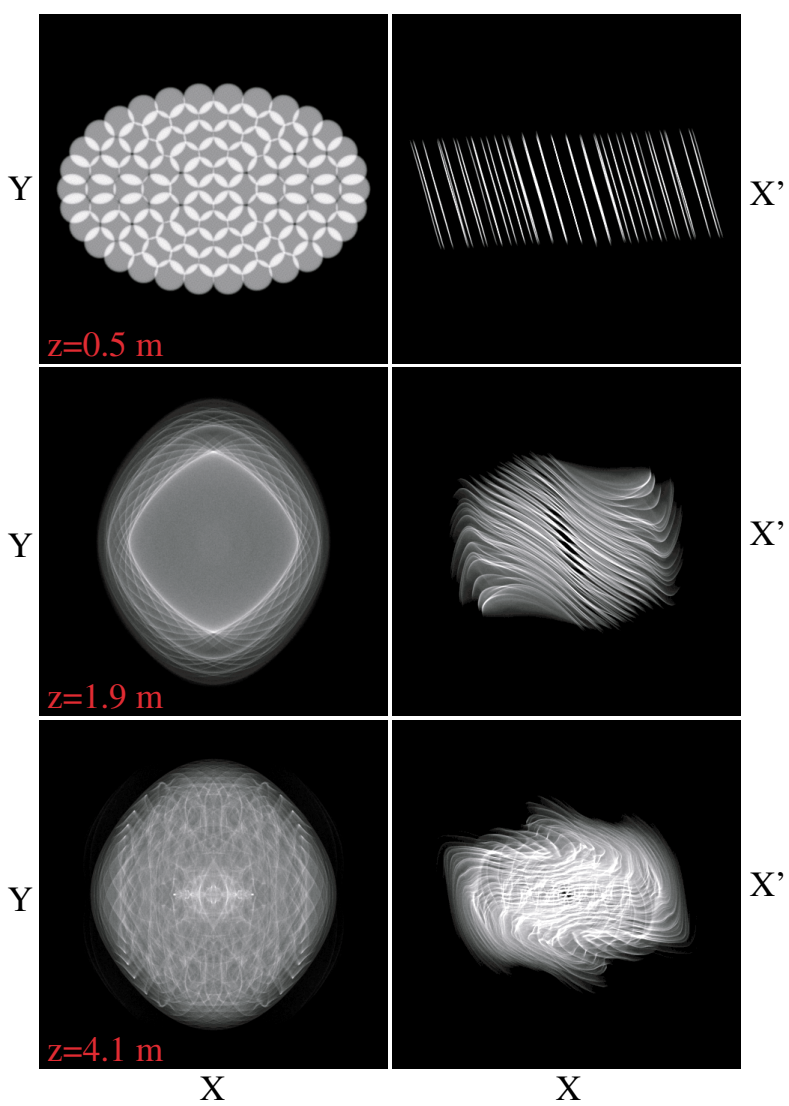

FIG. 4. (Color) Density plots of $(x-y)$ and $\left(x-x^{\prime}\right)$ spaces. In the $\left(x-x^{\prime}\right)$ plots, the gray scale is brightened. All densities greater than one-quarter of the maximum are drawn in white. From there, the gray scale linearly goes down to zero. The $z$ is the distance from the end of the preaccelerator column. 


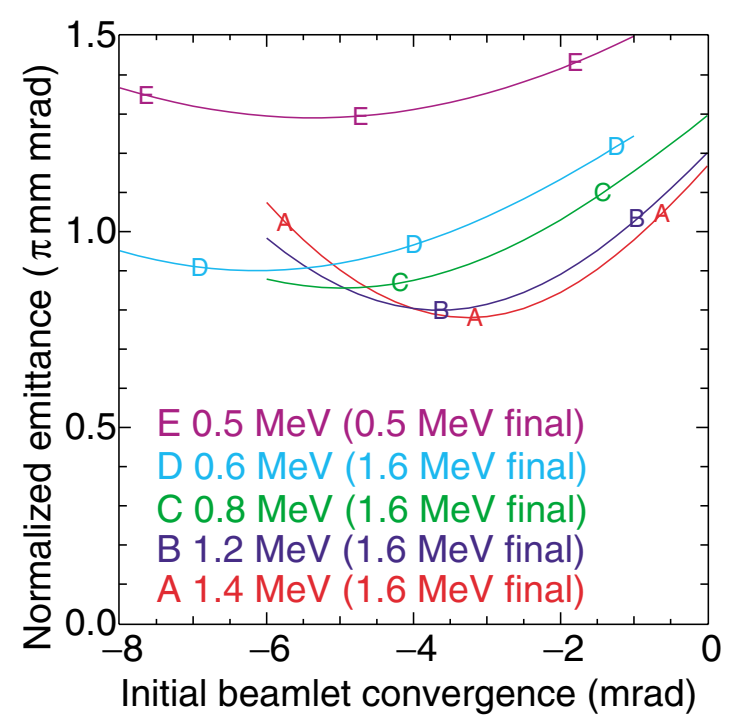

FIG. 5. (Color) Results from 2D slice simulations; emittance is shown versus the initial beamlet convergence angle. Compares cases with differing merging energies, for 91 beamlets and clearance $r_{\mathrm{c}}=0.5 \mathrm{~mm}$.

energy at which the beams are merged. Figure 5 shows the results of several series of simulations with varying merging energy. Keeping the configuration of beamlets emerging from the accelerating channel unchanged, the final emittance of the beam is seen to decrease with increasing merging energy as expected. The beamlet configuration is as determined above, but with a $0.5 \mathrm{~mm}$ clearance between the apertures of the last plate and the beamlet radius - the beamlet radii are $1.5 \mathrm{~mm}$. The length of the merging region decreased with decreasing merging energy. The case with merging at $0.5 \mathrm{MeV}$ is included as a possible design that might be used as a proof-of-principle experiment.

a)

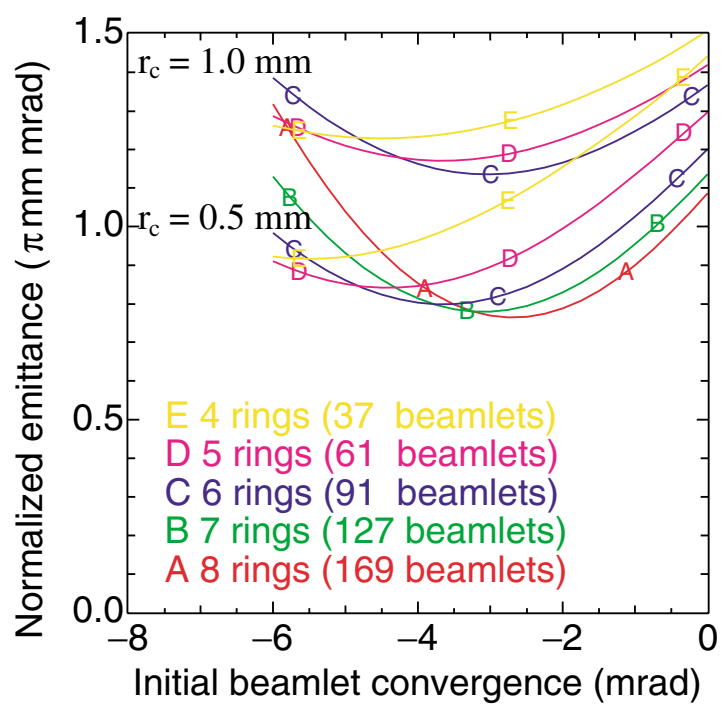

Figure 6 shows results with a varying number of beamlets. In Fig. 6(a), the number of beamlets is varied, holding fixed the initial charge density of the beamlets and $r_{\mathrm{c}}$, the clearance between the beamlets and the apertures (the difference in radii of the two). As the number of beamlets increases, the current per beamlet decreases, and therefore the beamlet size decreases. Holding the clearance fixed, the overall size of the configuration increases with an increasing number of beamlets. Note that cases with more than 91 beamlets have a larger footprint than the $7 \mathrm{~cm}$ limit. The emittance is seen to decrease with an increasing number of beamlets, as expected. With the charge density held fixed, the Einzel lens scaling, Eq. (4), gives a constant focusing strength. The initial temperature of the beamlets was taken to be $0.17 \mathrm{eV}$.

In Fig. 6(b), the number of beamlets was varied holding fixed the center-to-center beamlet spacing. To increase the number of beamlets, a new ring of beamlets was added to the outside of the configuration, increasing the overall size. Note that cases with fewer than 91 beamlets were not examined since the increased current per beamlet cannot be confined by the Einzel lenses.

These results show that there is quite a bit of flexibility in the design of the system without significant impact on the resulting emittance. The parameter with the most significant impact is the beamlet size. Increasing the number of beamlets gives only a small decrease in emittance as expected. Note also that in both cases, the width of the minimum becomes smaller with more beamlets. So, depending on how well the preaccelerator can actually be designed to give beamlet parameters near the minimum, the emittance advantage of more beamlets may further decrease.

Figure 7 shows the results from a series of simulations with varying beamlet size but with constant clearance, and with 91 beamlets merging at $1.2 \mathrm{MeV}$. The overall

b)

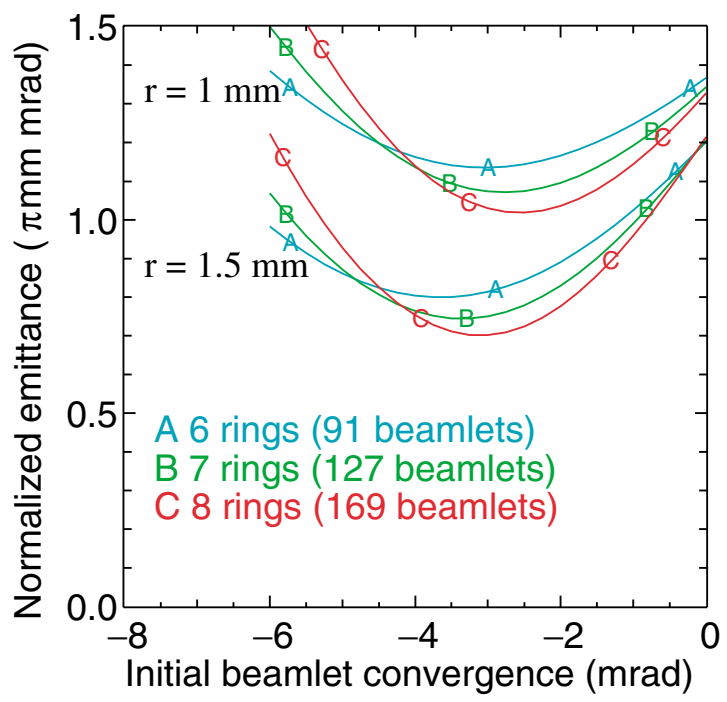

FIG. 6. (Color) Results from simulations with a differing number of beamlets. 


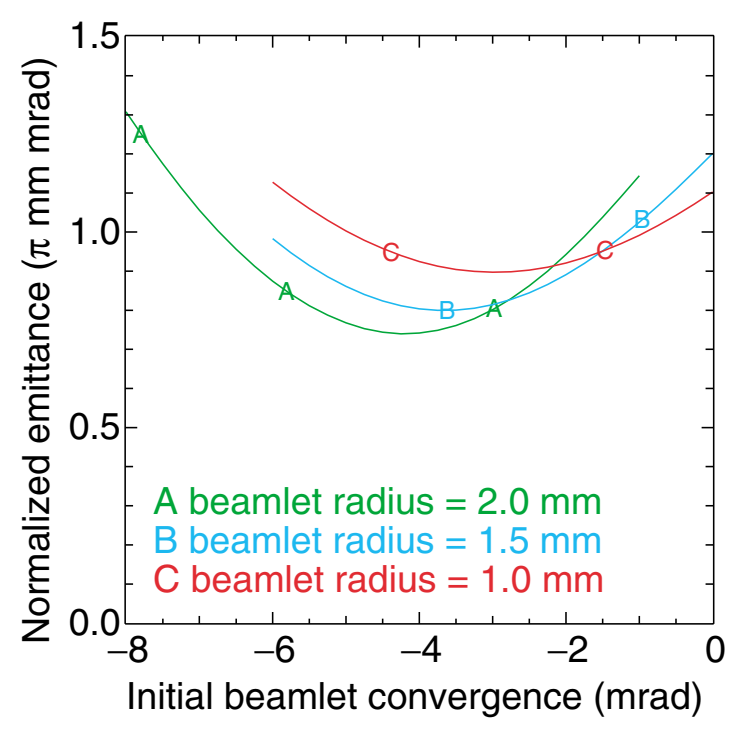

FIG. 7. (Color) The beamlet radii are varied while the clearance is held to a constant $0.5 \mathrm{~mm}$.

configuration size increases with increasing beamlet radii. This further shows the significance of the beamlet size on the emittance.

Also examined is the initial temperature (individual emittance) of the beamlets. Using the configuration as determined above ( 91 beamlets merging at $1.2 \mathrm{MeV}$ ), a series of simulations was done, varying the initial temperature of the beamlets. The final emittance, as shown in Fig. 8, is quite insensitive to the initial emittance of the beamlets. This allows much flexibility in the type of source used. For example, plasma sources, which typically have a higher operating temperature than other sources $(\geq 1.2 \mathrm{eV})$, but still within the range examined, could be used.

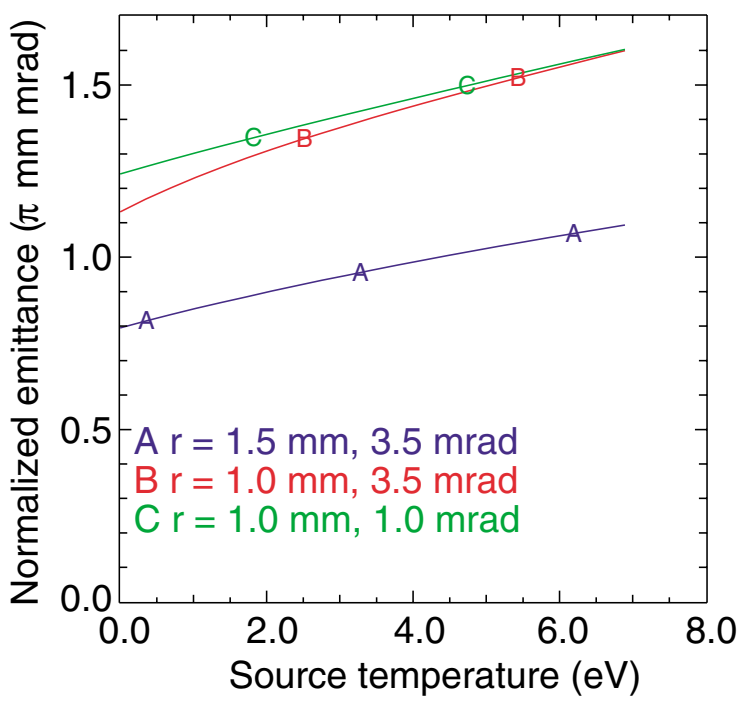

FIG. 8. (Color) Variation of final emittance with a range of initial source temperatures.

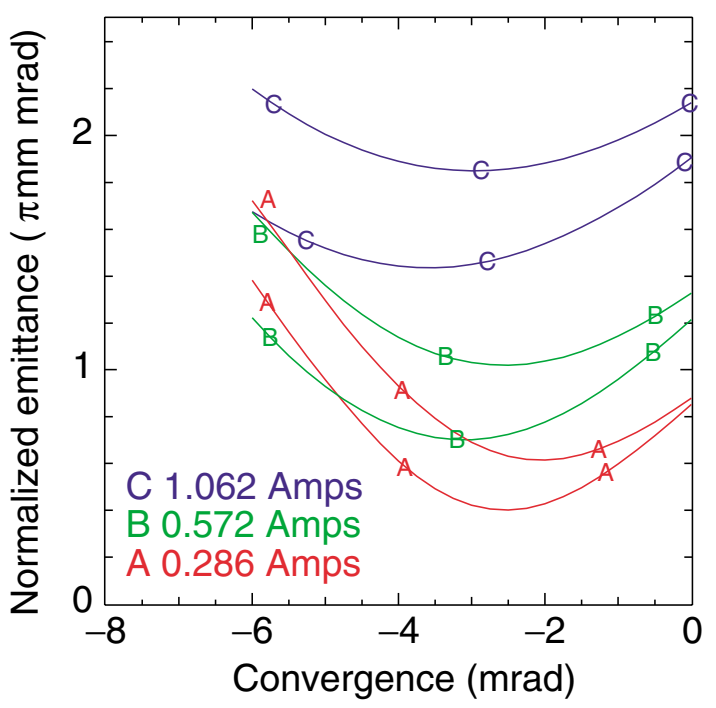

FIG. 9. (Color) Emittance versus convergence angle for various values of current, for the same number of beamlets, merging at 1.2 MeV. The upper curve in each pair is with beamlet radii of $1 \mathrm{~mm}$, and the lower is with $1.5 \mathrm{~mm}$.

Alternative designs of the accelerator may require a higher or lower current per beam. Figure 9 shows the scaling of the emittance versus convergence angle for different currents. Here, the total number of beamlets was held fixed (to 169, eight rings) and the current per beamlet was adjusted appropriately. The merged emittance is roughly proportional to the current. The brightness (current divided by emittance squared) then varies inversely proportional to the total current.

In order to examine sensitivities and tolerances in the design, transverse slice simulations of the merging region were carried out with errors in the initial position, aiming angle, size, and convergence of the beamlets. The primary measure of interest is the resulting beam emittance after the merge. In all cases, the emittance was found to be insensitive to the errors. With up to $0.7 \mathrm{~mm}$ errors in beam position and size, $0.4 \mathrm{mrad}$ in aiming angle, and $0.7 \mathrm{mrad}$ in beam convergence, the largest increase in emittance over the case with no errors was of the order of $20 \%$. It is expected that the errors in the accelerating column are all within that range. The effect of nonuniform density distribution in the beamlets was also examined. Distributions examined range from severely hollow (zero density on axis) to moderately peaked (50\% higher density on axis than edge). As with the other errors, the emittance was insensitive, varying only by $5 \%-10 \%$. Curiously, the emittance drops monotonically with increasing hollowness. The effect is small though, dropping only $10 \%$ for the most severely hollow beam.

\section{B. Preaccelerator}

In designing the preaccelerator, the same base case will be used as above, i.e., with 91 beamlets. Present-day 

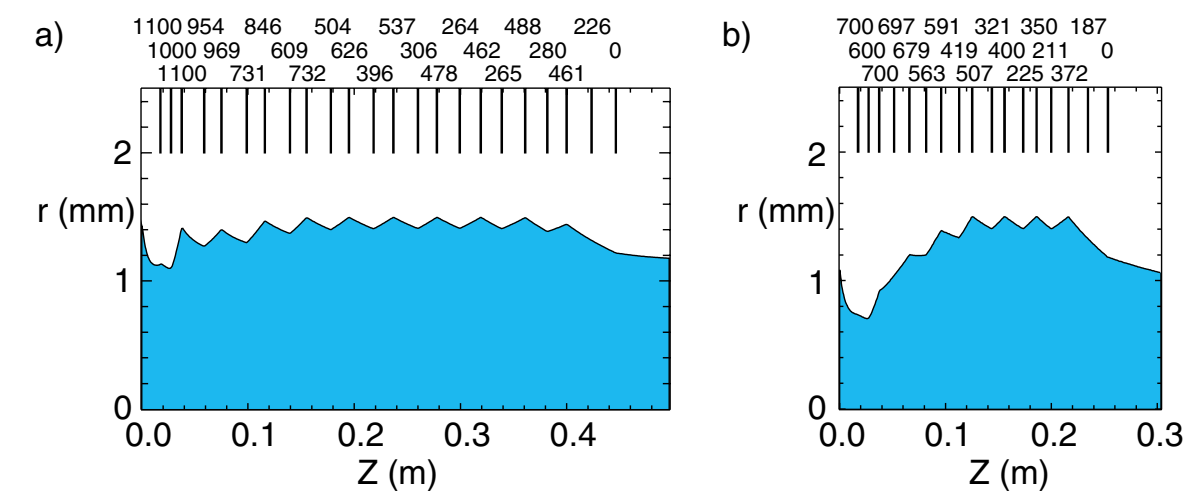

FIG. 10. (Color) Example layout of the aperture plates with the beam envelope. (a) Merging at $1.2 \mathrm{MeV}$ for a case with 91 beamlets. (b) Merging at $0.8 \mathrm{MeV}$ for a case with 169 beamlets. Both cases are well optimized. The voltages of the plates are given in $\mathrm{kV}$.

sources can emit reasonably well on the order of $100 \mathrm{~mA} / \mathrm{cm}^{2}$ [9]. To produce the specified current per beamlet at that current density, sources of radius $1.414 \mathrm{~mm}$ are required. See Fig. 10 for example layouts of the aperture plates for the $1.2 \mathrm{MeV}$ and $0.8 \mathrm{MeV}$ cases.

In designing the preaccelerator, there is a balance between getting a large beamlet size and getting the convergence angle close to the optimum (minimizing the emittance). The design becomes more constrained when including clearance between the beamlet and aperture, which is needed to allow for beamlet movement off axis due to construction errors. In the design shown in Fig. 10(a), the beamlet size at the exit of the column is $1.22 \mathrm{~mm}$ and the convergence is $-1.19 \mathrm{mrad}$ (i.e., converging), which is near the optimal value for this case. The merged emittance is estimated to be $1.11 \pi \mathrm{mm}$ mrad. Given the limited focusing strength of the Einzel lenses, it appears that the convergence cannot be further optimized without decreasing the beamlet size, and vice versa. Going to higher merge energy may improve the design, since the optimal convergence angle decreases with merge energy (see Fig. 5), but the focusing gets weaker at the end of the column and so the achievable convergence angle decreases.

To optimize the preaccelerator design, the multidimensional functional minimization algorithm Simultaneous Perturbation Stochastic Approximation was used [10]. The distances between the plates and the voltages on the plates were allowed to vary (except for the first three plates which were held fixed). This gave either 28 or 40 parameters (for 14 or 20 plates varied) for the two cases with the merging energies of 0.8 and $1.2 \mathrm{MeV}$. The calculation was done using an envelope solver and includes the focusing from the Einzel lenses and the acceleration. The field of the first three plates is obtained from a 3D Laplace solution. For the rest of the plates, the radial field given by the paraxial approximation is used.

$$
E_{r}=0.5 r\left(E_{z}^{-}-E_{z}^{+}\right) / l .
$$

Here, $r$ is the beamlet radius, $E_{z}^{-}$is the axial field to the left of the plate, $E_{z}^{+}$is the field to the right, and $l$ is an arbitrary length of the lens, taken in the calculation here to be the width of the plates, $1 \mathrm{~mm}$. The acceleration between plates is taken to be uniform. The axial fields are calculated knowing the voltage drop between plates and the plate separation. The envelope solution is initiated at the first plate using parameters obtained from steadystate particle simulations. Note that in the particle simulations, the emitter radius needed to be adjusted to obtain the desired current in each case since the injected current density was affected by the emitter radius. For the envelope calculation, the first three plates are held fixed so that the starting values from the particle simulation would be fixed.

Transverse slice (2D) particle simulations were used to obtain the final merged emittance as a function of the beamlet radii and convergence angles, and polynomial fits were made of the data. Using the fits, the merged emittance then could be estimated given the final beamlet size and convergence from the envelope solution of the preaccelerator. The optimization minimized this estimated emittance, while maintaining a clearance of $0.5 \mathrm{~mm}$ between beamlet and aperture and limiting the voltage gradients. (The reasons for the choice of $0.5 \mathrm{~mm}$ for the clearance are discussed in the next section.) Also, while the locations of the plates were allowed to vary for flexibility, the optimizer minimized the rms average of the plate separation, leading to nearly uniform plate spacing. The results from several optimized designs are shown in Table II. Note that the designs are not necessarily completely optimized, but are close. For example, the plate locations are not exactly evenly spaced.

The results show that, given the constraints, the merging beamlet configuration can achieve a normalized emittance near or below $1 \pi \mathrm{mm}$ mrad. There is little difference in the emittances with the differing merging energies. Even though the emittance will be lower with higher merging energy given the same beamlet size and 
TABLE II. Optimized aperture columns. Two cases were examined, with differing limits on the voltage gradients. In the first case (a), the gradient is independent of the plate separation. In the second case (b), the gradient depends on the plate separation, $d$, given here in centimeters.

\begin{tabular}{|c|c|c|c|c|c|c|c|c|}
\hline \multirow[b]{2}{*}{ \# Beamlets } & \multirow[b]{2}{*}{$\begin{array}{l}\text { Source radius } \\
\qquad(\mathrm{mm})\end{array}$} & \multirow[b]{2}{*}{$\begin{array}{c}\text { Merge energy } \\
(\mathrm{MeV})\end{array}$} & \multicolumn{3}{|c|}{ (a) $\max E z=100 \mathrm{kV} / \mathrm{cm}$} & \multicolumn{3}{|c|}{ (b) $\max E z=100 \mathrm{kV} / \mathrm{cm} \times d^{2 / 3}$} \\
\hline & & & $\begin{array}{c}a \\
(\mathrm{~mm})\end{array}$ & $\begin{array}{c}a^{\prime} \\
(\mathrm{mrad})\end{array}$ & $\begin{array}{c}\varepsilon_{\mathrm{n}} \\
(\pi \mathrm{mm} \operatorname{mrad})\end{array}$ & $\begin{array}{c}a \\
(\mathrm{~mm})\end{array}$ & $\begin{array}{c}a^{\prime} \\
(\operatorname{mrad})\end{array}$ & $\begin{array}{c}\varepsilon_{\mathrm{n}} \\
(\pi \mathrm{mm} \operatorname{mrad})\end{array}$ \\
\hline \multirow[t]{2}{*}{91} & 1.454 & 1.2 & 1.22 & -1.19 & 1.11 & 1.35 & 1.00 & 1.41 \\
\hline & & 0.8 & 1.16 & -2.33 & 1.17 & 1.20 & 0.04 & 1.39 \\
\hline \multirow[t]{2}{*}{127} & 1.125 & 1.2 & 1.18 & -1.70 & 1.01 & 1.14 & -0.79 & 1.15 \\
\hline & & 0.8 & 1.18 & -3.07 & 1.04 & 1.13 & -1.57 & 1.19 \\
\hline \multirow[t]{2}{*}{169} & 1.099 & 1.2 & 1.23 & -2.02 & 0.89 & 1.18 & -0.76 & 1.10 \\
\hline & & 0.8 & 1.18 & -2.80 & 0.99 & 1.10 & -1.86 & 1.12 \\
\hline
\end{tabular}

convergence, the column design is able to get closer to the optimum for the lower energy case since the focusing is stronger at the end of the column. The cases with additional rings were included to examine what advantage could be gained by increasing the configuration size beyond the footprint limit. Note that the center-to-center spacing was held fixed, so the outer radius increases with the square root of the number of beams.

\section{Preaccelerator errors}

Various errors were applied to the preaccelerator, such as voltage errors, transverse and longitudinal aperture offsets, and aperture radius errors. For each type of error, a large ensemble of calculations with differing random seeds was done for a range of magnitudes of maximum errors. Each individual error was chosen from a uniform random distribution within the range of plus/minus the maximum error. The beamlet was found to be fairly insensitive to all but the transverse aperture offsets. With a maximum error of $700 \mu \mathrm{m}$ in the longitudinal plate location and aperture radius, and a maximum error of $7 \mathrm{kV}$ in the plate voltages, the beamlet size, convergence, and emittance did not vary by more than $15 \%$, and in no cases were particles lost to the aperture plates.

With aperture offset errors, the beamlet is kicked off axis, leading in some cases to partial or complete beam loss. Figure 11(a) shows twice the rms beamlet offset taken over each ensemble for three different maximum offsets. (Assuming that the beamlet offsets will follow a Gaussian distribution, the rms offset is equivalent to $\sigma$, the Gaussian width. So, the probability that the offset will fall within twice the rms value, $2 \sigma$, is $95.4 \%$.) The final values of the beamlet offsets are show in Fig. 11(b). These data were generated using an envelope and centroid equation solver. This gives good agreement with the particle code and is much faster, allowing a very large number of sample cases (with differing random seeds). This assumes that the offsets are small enough to scale linearly and that the beam does not hit an aperture. The field is calculated for each aperture plate with a small offset, and the dipole component is extracted and saved. Then, for each calculation, the dipoles are scaled by a random
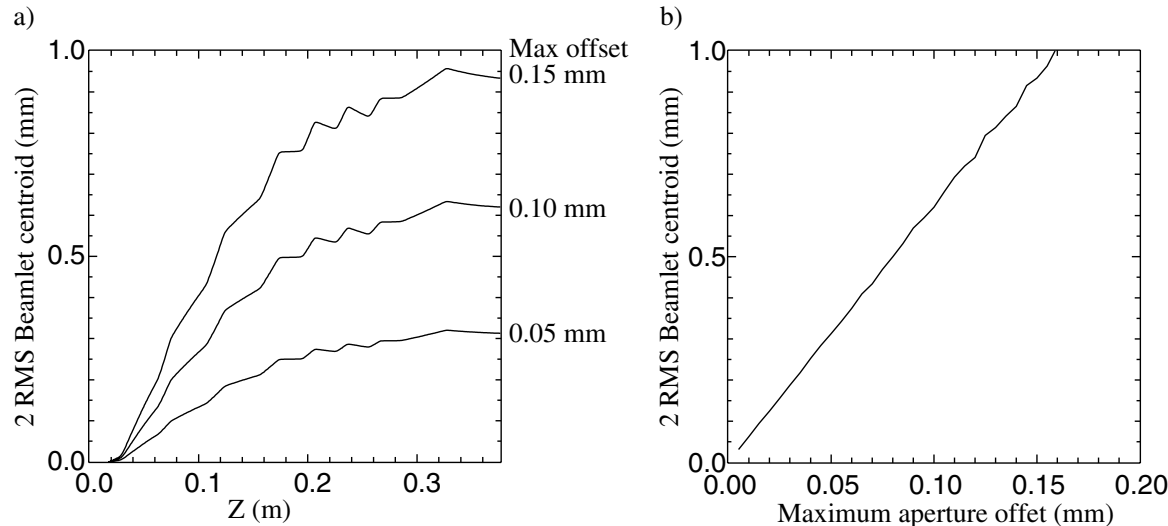

FIG. 11. Simulation results of a single beamlet channel with transverse aperture offsets. (a) Twice the rms average of each ensemble versus $z$. The offsets increase roughly with the square root of the number of elements. (b) Twice the final rms beamlet offset versus maximum aperture offset. 
offset. The focusing and acceleration components are assumed to be unchanged by aperture offsets.

A small number of cases were run with the threedimensional particle code for validation and to include emittance growth and particle scraping. For the cases simulated which had a clearance of $1 \mathrm{~mm}$ between the beamlet and the apertures, particle loss occurred with maximum offsets greater than $0.2 \mathrm{~mm}$. That result is less pessimistic than the envelope calculation which gives envelope excursions (centroid \pm envelope) greater than $2 \mathrm{~mm}$ for maximum offsets greater than about $0.15 \mathrm{~mm}$. In the cases with no particle loss, there was little effect on the emittance, no more than a $20 \%$ increase. Note also that the merged emittance was very insensitive to the beamlet emittance. Similarly, the beamlet size and convergence show little change, less than $10 \%$ variation. Figure 12 shows one sample case from the particle simulations, with $0.5 \mathrm{~mm}$ clearance and $0.2 \mathrm{~mm}$ maximum errors.

Design tolerances of the order of $100 \mu \mathrm{m}(0.1 \mathrm{~mm})$ are realistically achievable. With this tolerance, the order of
0.5 to $0.6 \mathrm{~mm}$ clearance will be required between the beamlets and the apertures. In the Einzel lenses used in these error studies, the beam was somewhat overfocused in the first half of the column as compared to the optimal designs in Sec. IIIB. Decreasing the focusing will relax the tolerances somewhat. So, the value of $0.5 \mathrm{~mm}$ was used for the clearance in the column optimization.

\section{ESTIMATE OF MERGED EMITTANCE}

An attempt was made to estimate the merged emittance using energy conservation. The initial energy of the arrangement of beamlets at the exit of the accelerating column (the point at which the beamlets interact) can be calculated either analytically or via the simulation as a sum of the kinetic energy and the field energy due to the space charge. An analytic expression for the space-charge field energy, $U_{\text {sc }}$, of $N$ nonoverlapping beamlets in the cylindrical pipe with radius $R$ can be derived [11].

$$
\begin{aligned}
U_{\mathrm{sc}}=\frac{1}{4 \pi \epsilon_{0}} & \left\{\sum_{i=1}^{N} \lambda_{i}^{2}\left[\ln \frac{a_{\mathrm{T}}}{a_{i}}+\ln \left(\frac{1-\delta_{i}^{2} / R^{2}}{1-\delta^{2} / R^{2}}\right)\right]-\sum_{j=1}^{N} \sum_{i<j} \lambda_{i} \lambda_{j}\left[\frac{1}{2}+\ln \left(\frac{\left|\delta_{i}-\delta_{j}\right|^{2}}{a_{\mathrm{T}}^{2}}\right)+\ln \left(1-\frac{\delta^{2}}{R^{2}}\right)^{2}-\ln \left(1-\frac{2 \delta_{i} \cdot \delta_{j}}{R^{2}}+\frac{\delta_{i}^{2} \delta_{j}^{2}}{R^{4}}\right)\right]\right. \\
& \left.+\frac{\sum_{i=1}^{N} \lambda_{i}^{2}}{4}\left(1+4 \ln \frac{R}{a_{\mathrm{T}}}\right)\right\} .
\end{aligned}
$$

Here, $\lambda_{i}, \delta_{i}$, and $a_{i}$, are, respectively, the line charge, centroid locations, and radii of the beamlets. The center of mass is

$$
\delta=\left(\sum_{i=1}^{N} \lambda_{i} \delta_{i}\right) / \lambda
$$

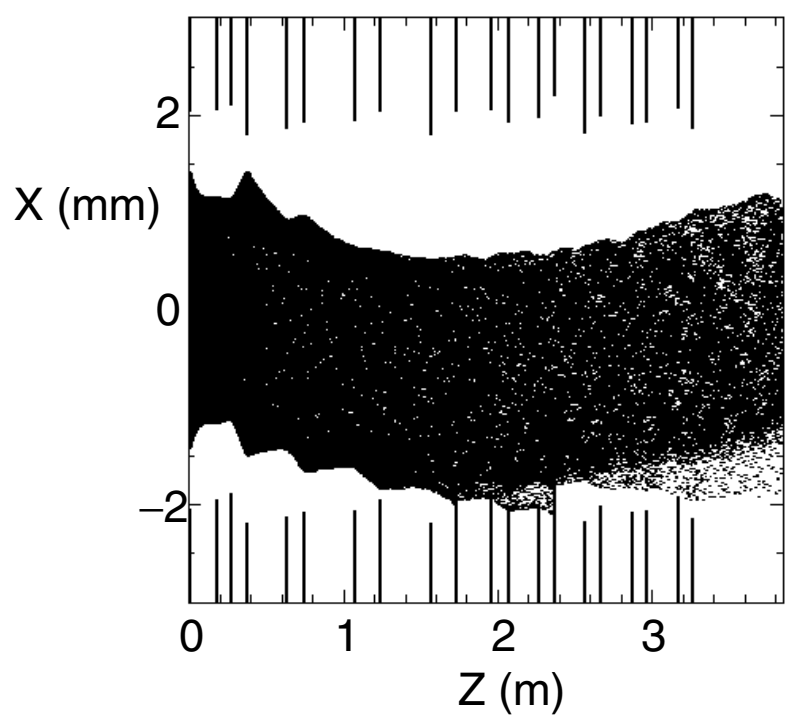

FIG. 12. Sample particle simulation showing the beamlet offset caused by aperture offsets. and the total beam size is

$$
a_{\mathrm{T}}^{2}=\left(\sum_{i=1}^{N} \lambda_{i}\left[a_{i}^{2}-2\left|\delta^{2}\right|+2\left|\delta_{i}^{2}\right|\right]\right) / \lambda,
$$

where $\lambda$ is the total line charge. For the cases described here, the center of mass $\delta$ is always zero and $a_{i}$, and $a_{i}^{\prime}$ (the beamlet convergence) are the same for all beamlets (written below without the subscript $i$ ). The kinetic energy of a single beamlet can be calculated by integrating $\frac{1}{2} m v^{2} f$ over the beamlet, where $f$ is the distribution function, $f=1 / \pi a^{2}$ inside the beamlet and zero outside, and $v^{2}=v_{z}^{2}\left(X^{\prime 2}+Y^{\prime 2}\right)$. The transverse velocities, $X^{\prime}$ and $Y^{\prime}$, each have two components, the convergence of the beamlet and the centroid motion, $X^{\prime}=x \frac{a^{\prime}}{a}+\delta_{i x} \frac{A^{\prime}}{A}$ and $Y^{\prime}=y \frac{a^{\prime}}{a}+\delta_{i y} \frac{B^{\prime}}{B}$, where $x$ and $y$ are the coordinates relative to the beam center, and $A, B, A^{\prime}$, and $B^{\prime}$, are the overall beam size and convergence given from the envelope solution. Note that for a round beamlet configuration, $A=B=a_{\mathrm{T}}$. The result of integration is summed over the $N$ beamlets to yield the total kinetic energy, $U_{\mathrm{ke}}$.

$$
U_{\mathrm{ke}}=\frac{\lambda_{i}}{2 q} m v_{z}^{2}\left\{\frac{1}{2} N a^{\prime 2}+\sum_{i=1}^{N}\left[\left(\delta_{i x} \frac{A^{\prime}}{A}\right)^{2}+\left(\delta_{i y} \frac{B^{\prime}}{B}\right)^{2}\right]\right\} \text {. }
$$

The normal procedure is to equate the initial and final energy, where for the final energy, the space-charge 
energy term is for a uniform beam and all of the kinetic part is assumed to be in the emittance [4]. There is a difficulty since the procedure relies on knowing what the matched beam radius is. The merged beam size produced by the simulation was typically smaller than the envelope solution by a few percent. The small difference had a large effect since the emittance is calculated by subtracting energies of similar magnitude. Using the larger beam size from the envelope gave an overestimate of emittance of a factor of 2 or more. Furthermore, the acceleration in the merging region complicates the procedure.

The following heuristic was found to produce a reasonably good estimate, even in the presence of acceleration. Assume that the "coherent" energy stays coherent, and the "incoherent" incoherent. The initial coherent energy is the sum of the space-charge energy from a uniform beam with equivalent $\mathrm{rms}$ size, $U_{\mathrm{sc}}^{\text {coherent }}$, and the kinetic energy is from the overall convergence, $U_{\mathrm{ke}}^{\text {coherent }}$. The space-charge energy of a uniform beam in a round pipe can be written as

$$
U_{\mathrm{sc}}^{\text {coherent }}=\frac{\lambda^{2}}{16 \pi \epsilon_{0}}\left(1+2 \ln \frac{2 R^{2}}{A^{2}+B^{2}}+2 \ln \frac{2\left(A^{2}+B^{2}\right)}{(A+B)^{2}}\right) .
$$

The coherent kinetic energy term can be written as

$$
U_{\mathrm{ke}}^{\text {coherent }}=\frac{\lambda}{2 q} m v_{z}^{2}\left(\frac{\left\langle X X^{\prime}\right\rangle^{2}}{\left\langle X^{2}\right\rangle}+\frac{\left\langle Y Y^{\prime}\right\rangle^{2}}{\left\langle Y^{2}\right\rangle}\right) .
$$

Here, $X$ and $Y$ are the coordinates, which can be written for each beamlet as $X=\delta_{i x}+x$ and $Y=\delta_{i y}+y$. The averages for each beamlet are

$$
\begin{gathered}
\left\langle X X^{\prime}\right\rangle_{i}=\frac{1}{4} a a^{\prime}+\delta_{i x}^{2} \frac{A^{\prime}}{A}, \\
\left\langle X^{2}\right\rangle_{i}=\frac{1}{4} a^{2}+\delta_{i x}^{2} .
\end{gathered}
$$

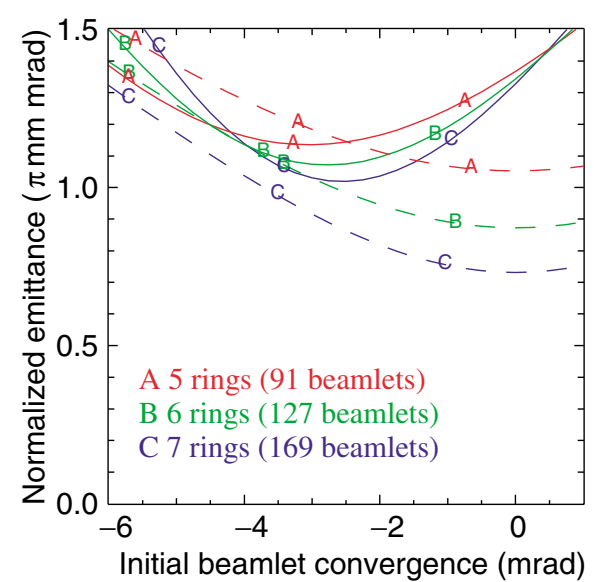

FIG. 13. (Color) Comparison of the estimated emittance and the results from the simulations. The solid curves are from the simulations, the dashed curves the estimates.
Averaging over the beamlets gives

$$
\begin{gathered}
\left\langle X X^{\prime}\right\rangle=\frac{1}{4} a a^{\prime}+\left|\delta_{i x}^{2}\right| \frac{A^{\prime}}{A}, \\
\left\langle X^{2}\right\rangle=\frac{1}{4} a^{2}+\left|\delta_{i x}^{2}\right|=\frac{1}{4} A^{2} .
\end{gathered}
$$

The coherent kinetic energy then evaluates to

$$
\begin{aligned}
U_{\mathrm{ke}}^{\text {coherent }}=\frac{2 \lambda}{q} m v_{z}^{2} & {\left[\frac{1}{A^{2}}\left(\frac{a a^{\prime}}{4}+\left|\delta_{i x}^{2}\right| \frac{A^{\prime}}{A}\right)^{2}\right.} \\
& \left.+\frac{1}{A^{2}}\left(\frac{a a^{\prime}}{4}+\left|\delta_{i y}^{2}\right| \frac{B^{\prime}}{B}\right)^{2}\right] .
\end{aligned}
$$

Furthermore, the $a a^{\prime}$ terms are dropped since they result from the convergence of the individual beamlets:

$$
U_{\mathrm{ke}}^{\text {coherent }}=\frac{2 \lambda}{q} m v_{z}^{2}\left[\frac{1}{A^{2}}\left(\left|\delta_{i x}^{2}\right| \frac{A^{\prime}}{A}\right)^{2}+\frac{1}{A^{2}}\left(\left|\delta_{i y}^{2}\right| \frac{B^{\prime}}{B}\right)^{2}\right] .
$$

After merging, all of the coherent energy goes into the space-charge energy. Assuming a round, uniform merged beam, Eq. (10) can be used to give the final space-charge energy. Setting $A$ and $B$ equal to $a_{\text {final }}$, the final beam radius can then be calculated.

$$
a_{\text {final }}=R \exp \left[-\frac{1}{4}\left(U_{\text {init }}^{\text {coherent }} \frac{16 \pi \varepsilon_{0}}{\lambda^{2}}-1\right)\right] .
$$

The remaining energy is incoherent and is all in the emittance, which can be found by writing the incoherent part of the kinetic energy (with the assumption that the $x$ and $y$ emittances are the same) as

$$
\begin{aligned}
U_{\mathrm{ke}}^{\text {incoherent }} & =\frac{\lambda}{4 q} m v_{z}^{2} \frac{\varepsilon_{x}^{2}}{a_{\text {final }}^{2}} \\
& =U_{\text {init }}^{\text {total }}-\left(U_{\mathrm{sc}}^{\text {coherent }}+U_{\mathrm{ke}}^{\text {coherent }}\right) .
\end{aligned}
$$

Here, $\varepsilon_{x}$ is the unnormalized emittance.

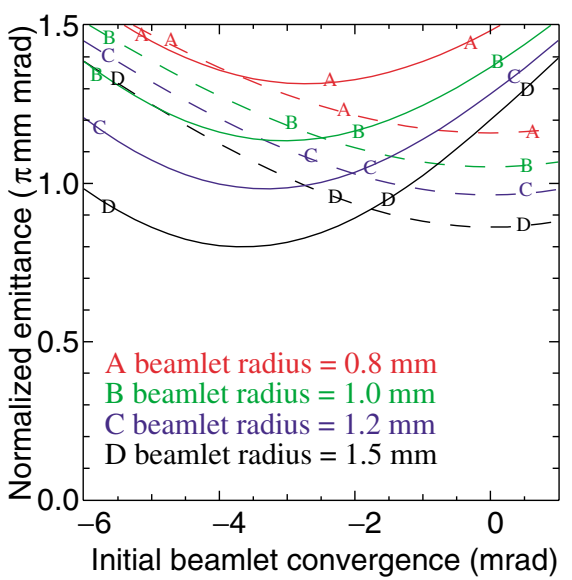


Results from this estimate are shown in Fig. 13. The estimate is consistently low and gives a minimum at a different location, but is close enough to be useful for rough scaling.

\section{CONCLUSION}

Given the results of these scaling studies, it appears that a multibeamlet injector can be built with a normalized emittance less than $1 \pi \mathrm{mm}$ mrad. For the parameters required in "an intermediate term facility for heavy-ion fusion" system, the case with 91 beamlets merging at $0.8 \mathrm{MeV}$ appears close to optimal. This design gives an emittance close to the value of $1 \pi \mathrm{mm}$ mrad. Increasing the merging energy does not give a significant decrease in the emittance due to the weakening focusing of the Einzel lenses. With other things being equal, the $0.8 \mathrm{MeV}$ design is preferable over the $1.2 \mathrm{MeV}$ simply because it uses fewer plates, simplifying construction. Merging at significantly less than $0.8 \mathrm{MeV}$ is less desirable since the voltage gradient in the subsequent accelerating region exceeds voltage breakdown limits.

For this design to work, the construction requires tight error tolerances, less than $0.1 \mathrm{~mm}$ error in the aperture location. If smaller errors are achievable, some reduction in emittance could be obtained by increasing the beamlet size, increasing the beamlet convergence angle, and/or decreasing the center-to-center spacing.

There are several additional areas that need further research. Little study has been done of the time-dependent behavior of the beam in the system. The rise time of the voltage on the column greatly affects the shape of the beamlet head. It will be important to resolve how the beamlet head can be made to travel through the channel with minimal particle loss to the apertures. Another issue is the strength of the interbeamlet forces in the column, including shielding by the aperture plates. Initial simulations (not reported here) show that this effect is small, but this needs closer examination. One other issue is the role of stray electrons, especially if any ions hit an aperture. It is expected that electrons will be swept out of the beamlets since the beamlet potentials are small. This can only be fully resolved via experiment.

\section{ACKNOWLEDGMENTS}

This work was performed under the auspices of the U.S. Department of Energy by the University of California, Lawrence Livermore, and Lawrence Berkeley National Laboratories under Contracts No. W-7405-Eng-48 and No. DE-AC03-76SF00098.

*Electronic address: DPGrote@lbl.gov

[1] J.W. Kwan, F. M. Bieniosek, E. Henestroza, L. Prost, and P. Seidl, "A 1.8 MeV K$K^{+}$Injector for the High Current Beam Transport Experiment," in Proceedings of the 14th International Symposium on Heavy-Ion Inertial Fusion, Moscow, Russia, 2002 [Laser Part. Beams (to be published)].

[2] High Voltage Technology, edited by L. L. Alston (Oxford University Press, New York, 1968), p. 65.

[3] L. Cranberg, J. Appl. Phys. 23, 518 (1952).

[4] O. A. Anderson, Fusion Eng. Des. 32-33, 209-217 (1996).

[5] J.W. Kwan, O. A. Anderson, D. N. Beck, F. M. Bieniosek, C. F. Chan, A. Faltens, E. Henestroza, S. A. MacLaren, P. A. Seidl, L. Ahle, D. P. Grote, E. Halaxa, and C.T. Sangster, in Proceedings of the 1999 Particle Accelerator Conference, New York (IEEE, Piscataway, NJ, 1999), p. 1937.

[6] J.W. Kwan, L. Ahle, D. N. Beck, F. M. Bieniosek, A. Faltens, D. P. Grote, E. Halaxa, E. Henestroza, W. B. Herrmannsfeldt, V. Karpenko, and T. C. Sangster, Nucl. Instrum. Methods Phys. Res., Sect. A 464, 379-387 (2001).

[7] D. P. Grote, A. Friedman, I. Haber, W. Fawley, and J. L. Vay, Nucl. Instrum. Methods Phys. Res., Sect. A 415, 428-432 (1998).

[8] I. Haber, D. Kehne, M. Reiser, and H. Rudd, Phys. Rev. A 44, 5194 (1991).

[9] L. Ahle, R. Hall, A. Molvik, E. Chacon-Golcher, J.W. Kwan, K. N. Leung, and J. Reijonen, in Proceedings of the Particle Accelerator Conference, Chicago, IL, 2001 (IEEE, Piscataway, NJ, 2001), p. 726.

[10] J. C. Spall, IEEE Trans. Autom. Control 37, 332 (1992).

[11] E. P. Lee (private communication). 